\title{
ADAPTACIÓN CULTURAL DE LA ESCALA PARA MEDIR CONOCIMIENTO SOBRE HERPES SIMPLE GENITAL EN ADULTOS JÓVENES CUBANOS
}

\author{
CROSS CULTURAL ADAPTATION OF THE SCALE TO \\ ASSESS KNOWLEDGE ABOUT GENITAL HERPES
}

\author{
Abdul Hernández Cortina * \\ Erika Caballero Muñoz ${ }^{* *}$
}

\begin{abstract}
RESUMEN
Objetivo: Adaptar culturalmente al contexto cubano la escala para medir conocimiento sobre herpes simple genital. Material y métodos: El proceso de adaptación cultural de la escala incluyó la realización de traducción, retro-traducción, revisión por un comité de expertos, preprueba y envío de la escala adaptada a los autores originales. El estudio fue realizado de junio a noviembre del año 2008 con 38 estudiantes de licenciatura en enfermería matriculados en la Escuela de Enfermería de San Antonio de los Baños, Habana, Cuba. Los permisos de la institución y el comité de ética de la facultad fueron obtenidos antes de comenzar el estudio. Resultados: El $53 \%$ fueron mujeres $(n=17)$ y el $47 \%$ fueron hombres $(n=18)$. El $84 \%$ de la muestra estaba soltero $(n=32), 13 \%$ casados $(n=5)$ y $2,6 \%$ estaban divorciados $(n=1)$. Todos los integrantes de la muestra estaban estudiando licenciatura en enfermería $(n=38)$. La estabilidad interna del instrumento con el coeficiente de Alfa de Cronbach fue de 0.82 y el Coeficiente de Correlación Interclase (ICC) fue de 0.84. Conclusiones: La escala es confiable en su uso para medir conocimiento sobre herpes simple genital en el contexto cubano. Por lo tanto la escala para medir conocimiento sobre herpes simple genital es un instrumento apropiado para realizar investigaciones en el área de las infecciones de transmisión sexual en adultos jóvenes.
\end{abstract}

Palabras clave: Herpes genital, psicometría, infecciones de transmisión sexual, enfermedades genitales.

\begin{abstract}
Objective: The aim of this manuscript is to adapt culturally to the Cuban context the scale to measure knowledge about genital herpes simplex. Methods: A cross-sectional design was used with an exploratory survey methodology from June 2008 to November 2008 with nursing students from the school in San Antonio de los Baños, Havana Cuba. Institutional and ethical approval was obtained before starting the study. Results: The mean age of the participants was 23.34 years with (SD 7.01), and $53 \%(n=17)$ were female and $47 \%$ were male $(n=18) .84 \%$ of student were unmarried $(n=32), 13 \%$ were married $(n=5)$ and $2,6 \%$ were divorced $(n=1)$. All subjects were involved in the bachelor's program in nursing $(n=38)$. The Alpha Coefficient for the total scale was 0,82 . The interclass correlation coefficient to measure scale's stability over time was 0,84 (test-retest two weeks). Conclusions: Findings support that genital herpes knowledge scale is a reliable and valid in measuring knowledge young adults. Thus, genital herpes knowledge scale is an appropriate instrument to conduct the research on sexual transmitted disease in young adults in the Cuban contexts.
\end{abstract}

Key words: Herpes genitalis, psychometrics, sexually transmitted disease, genital diseases.

Fecha recepción: 20/12/10 Fecha aceptación: 20/11/12

\footnotetext{
* Enfermero. Docente Instituto Superior de Ciencias Médicas de la Habana. E-mail: adher73@yahoo.es, adher@eictv.org.cu ** Enfermera matrona. Directora del Centro de Informática en Salud de la Universidad Central. Santiago de Chile. Chile. E-mail: ecaballe@gmail.com
} 


\section{INTRODUCCIÓN}

Las infecciones de transmisión sexual (ITS) $y$ el virus de inmunodeficiencia humano causante del síndrome de inmunodeficiencia humana VIH/SIDA constituyen un problema de salud en países latinoamericanos. Cada año su incidencia y prevalencia aumenta a pesar de las medidas adoptadas por los sistemas de salud de los diferentes países. Entre las ITS que constituyen un problema actual de salud está el herpes simple genital. Debido a su cronicidad, su carácter recurrente y su transmisibilidad, esta enfermedad se ha situado entre las de mayor incidencia y prevalencia en Latinoamérica y el Caribe (1-4). Por tal motivo la necesidad de dirigir intervenciones a las poblaciones con riesgo de contraer herpes simple genital así como a las personas que lo padecen ha sido manifestada por diferentes autores (59). De forma general los programas para la atención de las personas con ITS-VIH/SIDA han sido dirigidos a incrementar el uso del condón masculino $(10,11)$, el sexo seguro $(12,13)$ y aumentar el conocimiento sobre la enfermedad $(14,15)$.

El conocimiento sobre el herpes simple genital en las personas con alto riesgo de adquirirlo y en los pacientes que la padecen disminuye la transmisión de la enfermedad, mejora el estado de ánimo e incrementa la autoeficacia para hacer frente a la patología. Estas ventajas han sido reconocidas en estudios sobre el tema. Por ejemplo, Nath y Thappa (16) señalaban la importancia de brindar información sobre la enfermedad en pacientes con reciente diagnóstico. Mertz (17) recomendaba informar sobre la transmisibilidad, los tratamientos antivirales y el uso del condón en los pacientes con diagnóstico clínico de herpes simple genital. Warren y Ebel (18) consideraban pertinente compartir información con los pacientes durante la consejería como factor elemental en la reducción de la ansiedad y el estrés. Dibble y Swanson (19) evaluaron la autoeficacia de pacientes con herpes simple genital después de tres sesiones en las cuales se brindaba información sobre la afección.

En Cuba el herpes simple genital se encuentra entre las enfermedades de declaración obligatoria del Ministerio de Salud Pública, siendo una de las líneas prioritarias dentro del programa de atención a las ITS/ VIH/ SIDA. A nivel comunitario es la enfermera quien asume las funciones de promoción y prevención de estas patologías de transmisión sexual. Por lo tanto, para conducir la práctica y la investigación a nivel comunitario se necesita de escalas que permitan recoger y validar datos seguros y confiables para la toma de decisiones en el cuidado de la persona con infecciones de transmisión sexual. Sin embargo, en la revisión de la literatura realizada en las bases de datos EBSCHO, PubMED y CINAHL no se encontraron escalas validadas para el contexto latinoamericano que midan conocimiento sobre el herpes simple genital. No obstante, se encontró una escala para medir conocimiento sobre herpes simple denominada Herpes Knowledge Scale (HKS) realizada por Bruce y MacLaughlin en la Facultad de Psicología de la Universidad de North Carolina en los Estados Unidos en 1986 (20). Esta escala aborda la totalidad de la información que deben conocer los adultos jóvenes con riesgo a padecer la enfermedad y los que ya la padecen. Pero necesita ser validada en el contexto cubano para su uso confiable. Por esta razón se planteó la siguiente interrogante científica:

¿La escala para medir conocimiento sobre herpes simple genital es confiable para su uso en investigaciones en el contexto cubano?

Para ello nos trazamos como objetivo: adaptar culturalmente al contexto cubano la escala para medir conocimiento sobre herpes simple genital. 


\section{MATERIAL Y MÉTODOS}

Este es un estudio donde se realizó una adaptación cultural en estudiantes de enfermería de 5to año de la Universidad de Ciencias Médicas de la Habana. Para el proceso de adaptación cultural de la escala se tomó como referente la metodología propuesta por Guillemin, Bombardier \& Beaton (21), que plantea cinco escenarios, que relacionan la cultura de la población diana, el lenguaje del instrumento y el país de utilización.

Este estudio corresponde al quinto escenario, otra cultura, otro lenguaje y otro país. Los autores recomiendan cinco pasos para la adaptación cultural: Traducción, retro-traducción, revisión por un comité de expertos, preprueba y envío de la escala adaptada a los autores originales (21). La traducción se realizó por el Servicio de Traductores e Intérpretes en Cuba (ESTI). La retro-traducción fue realizada por dos profesores de idioma inglés con más de 20 años de experiencia en la formación de estudiantes de medicina y enfermería. Para la revisión por comité de expertos se utilizó la metodología de Moriyama (22) en la cual se valora la pertinencia de los ítemes basado en cinco criterios a saber: razonable y comprensible, sensible a variaciones, con suposiciones básicas justificables e intuitivamente razonables, con componentes claramente definidos, y derivable de datos factibles de obtener. Posteriormente se aplicó la escala a una muestra de 38 estudiantes de enfermería de la filial de San Antonio de los Baños previo consentimiento informado, autorización por parte de la jefa de carrera y aprobación por el comité de ética de la facultad. La escala se aplicó dos veces a los mismos estudiantes en un intervalo de 15 días para calcular su estabilidad en el tiempo. Para el análisis estadístico se calcularon las medias en la variable cuantitativa discreta y porcentajes para las cualitativas nominales politómicas y dicotómicas. También se calculó el coeficiente Alfa de Cronbach que mide la homogeneidad y el coeficiente de correlación interclase para medir la estabilidad en el tiempo de la escala.

Esta escala autoaplicada para medir conocimiento sobre herpes simple está formada por 54 ítemes. Sus opciones de respuesta son tres: verdadero, falso y no conozco la respuesta. Las respuestas correctas acumulan un punto y las incorrectas y no conozco la respuesta no acumulan puntos. A la sumatoria de los puntos se le calcula el porcentaje (rango de 0 a 100\%). A mayor porcentaje de respuestas correctas mayor conocimiento sobre herpes simple. Esta escala se contesta aproximadamente en $15 \mathrm{~min}$.

\section{RESULTADOS}

\section{Retro-traducción}

En los resultados de la retro-traducción se cambiaron las palabras "longitud" y "gravedad" en el ítem 1, por los términos "duración" y "severidad", debido a que estas palabras dan el significado real de lo que se pretendía preguntar. En el ítem 22 se sustituyó "hoy existe cura para los herpes genitales" por "existe cura para el herpes genital en estos momentos". De acuerdo con los profesores de inglés la oración sustituida ofrece al lector una mejor orientación en el tiempo y evita la confusión, si es que en el lugar no hay medicamento para curar o si es que no existe cura para la enfermedad de forma general. El ítem 25 también es sustituido pasando de: "Cuando el herpes genital debuta de manera agresiva es aconsejable no tener relaciones sexuales" por "Cuando la persona tiene las lesiones es recomendable no tener relaciones sexuales". Esta segunda opción es más sencilla y legible que la primera (Anexo 1).

Otro de los ítemes al que se le realizaron cambios fue el 28, en el cual es sustituido "Los herpes genitales pueden ser contagiosos 
aunque tengan una costra que los cubra" por "El herpes genital puede contagiarse aunque la lesión esté cubierta con una costra". La segunda oración ofrece mayor claridad según los profesores que realizaron la retro-traducción. El ítem 37 también fue modificado de "Los condones ofrecen un 100\% de protección contra el herpes genital" por "El uso del condón ofrece un $100 \%$ de protección contra el herpes genital". En la primera oración da a entender que los condones ofrecen protección por sí mismo y no el uso que le dé la persona.

La traducción del ítem 48 fue sustituida de "En un caso primario (primero) de herpes genital, la persona puede sentir que tiene catarro" por "En el primer episodio con herpes genital la persona puede sentirse como si tuviera catarro". La segunda opción ofrece mayor coherencia entre la primera idea (episodio de herpes genital) y la segunda idea (sentirse como si tuviera catarro). Otro de los ítemes que fueron cambiados fue el 50 . Se sustituyó "La mejor manera de combatir las llagas por herpes genital es mantenerlas húmedas" por "la mejor forma de curar la lesión del herpes simple genital es manteniendo la lesión húmeda”. La segunda opción sustituye el verbo de acción "combatir" por "curar". El verbo "curar" manifiesta de mejor manera el significado que se desea transmitir en el ítem.

\section{Revisión por un comité de expertos}

En los resultados de los criterios de expertos se modificaron algunos ítemes y otros fueron eliminados. En este sentido el ítem 7 no cumplió el criterio: con componentes claramente definidos, tres de los expertos sugirieron sustituir el término "neurona" por "células nerviosas" para que pudiera ser de mejor comprensión a las personas que responden el cuestionario. Lo mismo sucedió con el ítem 10. La pregunta hace referencia a las apariciones del herpes simple, la forma que se pregunta puede conllevar a confusiones debido a que los pacientes pueden tener descarga viral y no tener síntomas. O sea en la forma que se plantea este ítem no se distingue si se hace referencia a la aparición de la lesión o a las descargas virales que manifiestan los pacientes, y que son silentes para la mayoría de las personas que padecen la enfermedad. Según los expertos, la mejor forma de redactar esta pregunta es: "Pueden pasar años entre la aparición de las lesiones por herpes simple". En esta otra opción se describe con mayor claridad el conocimiento que se quiere medir.

El ítem 13 tampoco cumplió con el criterio: con componentes claramente definidos. Los cinco expertos consideraron que la pregunta no se expresaba claramente por causa del uso del término "llagas frías". Como consecuencia se modificó el ítem por: "Las personas que tienen lesiones por herpes en la boca son inmunes a adquirir las lesiones en los genitales". Otro ítem que fue modificado fue el 14 debido a que tres expertos consideraron que no cumplía con el criterio con componentes claramente definidos. En este sentido los expertos recomendaron modificar el término de "ampolla" por "vesículas agrupadas" ya que esa última constituía la característica clínica fundamental de la lesión por herpes simple genital.

Teniendo en cuenta el criterio de tres expertos se eliminó el ítem 27 ya que éstos consideraban que no cumplía con el criterio: con suposiciones básicas justificables e intuitivamente razonables. Los expertos alegaron que no se encontraban evidencias de que el herpes simple genital provocaba cáncer cervico uterino en las mujeres que lo padecían. Por lo tanto, en Cuba no se indicaba la prueba citológica anualmente en estos pacientes. Seguidamente el ítem 29 fue modificado ya que cuatro expertos consideraron que no cumplía con el criterio: con componentes claramente definidos. De esta forma se redactó de la siguiente forma: "La primera infección por herpes genital es más severa que las reapariciones". Se deci- 
dió sustituir la palabra "grave" por "severo". Este concepto ofrecía con mejor claridad la información que se quería reflejar en el cuestionario. Con este propósito, se buscaba medir el conocimiento sobre la severidad de las recurrencias y no sobre la gravedad de la enfermedad. Por otra parte en el ítem 34 los cinco expertos consideraron que no cumplía con el criterio: con componentes claramente definidos debido a que "frotis de PAP" no era el término que se utilizaba en nuestro medio, siendo el término de "prueba citológica". Por tal motivo se modificó este ítem a: "Con frecuencia el herpes genital suele detectarse mediante la prueba citológica”.

Por último, los ítemes 45 y 47 fueron eliminados ya que no cumplían con el criterio: razonable y comprensible. La mayoría de los expertos consideró que no se deben hacer referencias a los tratamientos ni a sus dosis en los instrumentos. Por otra parte en el contexto cubano no está disponible la espuma anticonceptiva para su uso en los pacientes. Por lo tanto no estaba justificada la inclusión de estas preguntas en el instrumento debido a que había una gran probabilidad de que no conocieran las respuestas.

\section{Pre-prueba}

Los resultados de la pre-prueba o estudio piloto fueron los siguientes. La media de edad de los participantes fue de 23.34 años con una desviación estándar de 7.01 años. El $53 \%$ fueron mujeres $(n=17)$ y el $47 \%$ fueron hombres $(n=18)$. El 84\% de la muestra estaba soltero $(n=32), 13 \%$ casados $n=5$ y $2.6 \%$ estaban divorciados $(n=1)$. Todos los integrantes de la muestra estaban estudiando licenciatura en enfermería $(n=38)$.

El puntaje medio correcto fue de $48 \%$ con un puntaje mínimo de $10 \%$ y un máximo de 86.4\%. La estabilidad interna del instrumento con el coeficiente de Alfa de Cronbach fue de 0.82 y el Coeficiente de Correlación Interclase (ICC) fue de 0.84 .

\section{Envío de la escala a los autores originales}

Se envió por e-mail la escala adaptada a los autores originales. Los mismos dieron su aprobación y consentimiento sobre el proceso realizado.

\section{DISCUSIÓN Y COMENTARIO}

La escala original posee 54 ítemes, mientras que la versión al español posee 51 ítemes. La eliminación de tres ítemes está sustentada por las diferencias culturales en cuanto a brindar información sobre las dosis de amino-glucósidos o no. De acuerdo con algunos autores en la consejería no se debe brindar información a los pacientes sobre el cálculo de las dosis o tratamientos para evitar la automedicación (23-25). En cuanto a la forma de medición, la escala adaptada se mantiene de la misma forma a la escala original. Un porcentaje elevado de la escala indica mayor conocimiento sobre la enfermedad. La consistencia interna del Gold Estándar Americano fue Alfa Cronbach 0.88 . El puntaje medio correcto de la escala adaptada fue menor si tenemos en cuenta que el puntaje del instrumento original fue de $64 \%$. El puntaje mínimo fue de $7.4 \%$ y el más alto fue de $92.6 \%$ (20). En la versión al español de la escala el resultado del Alfa Cronbach fue menor con un 0.82. La diferencia que existe entre el Gold Estándar Americano en los tests que miden homogeneidad (Alpha Cronbach) y los promedios de respuestas correctas puede ser debido a las diferencias culturales respecto al conocimiento sobre el herpes simple genital entre los jóvenes estadounidenses y los cubanos más que una distorsión de la escala resultante de la traducción. En este sentido algunos estudios y reportes manifiestan que el conocimiento sobre algunas ITS es bajo en poblaciones de alto riesgo en Cuba (26-31). En la versión original los autores no realizaron el cálculo de estabilidad en el tiempo de la escala. Sin embargo, en la versión al español el 
cálculo de estabilidad en el tiempo se realizó para determinar si los resultados de la escala eran diferentes en el transcurso del tiempo. Esto permite utilizar la escala en estudios experimentales donde se mide el conocimiento en intervalos de tiempo diferentes en una misma muestra. Por otra parte, en las revisiones de la literatura de enfermería latinoamericana no se encontró una escala similar que mida conocimientos sobre herpes simple genital que constituya un Gold Estándar de comparación con la escala sometida al proceso de adaptación cultural. El determinar conocimientos constituye un factor protector en la transmisión de la enfermedad, ya que permite empoderar al usuario con conocimientos que le orienten en su toma de decisiones correctas en cuanto a su conducta sexual y su relación con su pareja.

La adaptación cultural de la escala ha permitido contextualizar la forma de valorar los conocimientos del herpes simple genital. Es importante señalar que la enfermería como ciencia y disciplina requiere de instrumentos con las propiedades psicométricas que permitan recoger y validar datos seguros y confiables para tomar decisiones en el cuidado de la persona, familia y comunidad. En este sentido la escala es confiable y válida en su uso para medir conocimiento sobre herpes simple genital en el contexto cubano. Por lo tanto, la escala para medir conocimiento sobre herpes simple genital es un instrumento apropiado para realizar investigaciones en el área de las infecciones de transmisión sexual en adultos jóvenes. Lo que es pertinente de ser utilizado por enfermeras para estructurar en base a sus hallazgos un sistema psicoeducativo de apoyo a los pacientes con herpes simple genital.

\section{REFERENCIAS}

1. Looker KJ, Garnett PG, Schmid GP. An estimate of the global prevalence and incidence of herpes simplex virus type 2 in- fection. Public Health Rev. 2008; 86(10): 805-12.

2. Martínez MJ, Navarrete N, Santander E C, Garmendia M L, Gubelin M. Seropervalencia de la infección por virus herpes simplex tipo 2 en pacientes atendidos en centros de referencia de ETS de Santiago. Rev Med Chil. 2005; 133(3): 302-306.

3. Hernández CA. Infección por herpes simple. Revisión global. Rev. habanera cienc. Méd [Internet] 2008 Dic [citado 13 agosto 2010]; 7(3): [10 p.] Disponible en: http://www.ucmh.sld.cu/rhab/rhcm vol_7num_4/rhcm20408.htm

4. Smith SJ, Robinson J. Age Specific Prevalence of Infection with Herpes Simplex Virus Types 2 and 1: A Global Review. J Infect Dis [Internet] 2002 Oct [citado 20 abril 2007]; 186(1): [26 p.] Disponible en: http://www.journals.uchicago.edu.proxy1.lib.umanitoba.ca/doi/ full/10.1086/343739

5. Freeman EE, Weiss HA, Glynn JR, Cross PL, Whitworth JA, Hayes RJ. Herpes simplex virus 2 infection increases HIV acquisition in men and women: systematic review and meta-analysis of longitudinal studies. AIDS. 2006; 20: 73-83.

6. Watson-Jones D, Weiss HA, Rusizoka M, Changalucha J, Baisley K, Mugeye K, et al. Effect of herpes simplex suppression on incidence of HIV among women in Tanzania. N Engl J Med. 2008; 358(15): 1560-1571.

7. Nath AK, Thappa DM. Newer trends in the management of genital herpes. Indian J Dermatol Venereol Leprol. 2009; 75(6): 566-74.

8. Organización Mundial de la Salud. Preparing a health care workforce for the 21st century: the challenge of chronic conditions. Informe de la OMS. Ginebra: OMS; 2005.

9. Edward W, Hook PL. Time to Translate New Knowledge into Practice: A Call for a National Genital Herpes Control Program. J Infect Dis. 2006; 194(1): 6-7. 
10. Montijo SR, Arroyo BF, Cervantes MR, Barroso R, Villegas ED, Campuzano MR, et al. Entrenamiento conductual en habilidades de comunicación sexual en la pareja y uso correcto del condón. An Psicol. 2006; 22(1): 60-71.

11. Casey K, Timmermann L, Allen M, Krahn S, LaPlant K. Response and SelfEfficacy of Condom Use: A Meta-Analysis of this Important Element of AIDS Education and Prevention. Southern Communication Journal [Internet] 2010 Enr [citado 3 diciembre 2010]; 74 (1): [21 p.] Disponible en: http://dx.doi. org/10.1080/10417940802335953

12. Craig R. Genital herpes in young adults: Changing sexual behaviors, epidemiology and management. Herpes. 2005; 12(1): 10-14.

13. Australian Herpes Management Forum. Herpes transmission and safe sex [Internet]. Westmead Australia: Westmead Hospital; 1997-2010 [actualizado 20 octubre 2010; citado 24 noviembre 2010]. Disponible en: http://www.ahmf.com.au/

14. Wald A, Selke S, Lairson E, Rhoda AM, Zeh J. Knowledge of Partners' Genital Herpes Protects against Herpes Simplex Virus Type 2 Acquisition. J Infect Dis. 2006; 194(1): 42-52.

15. Onwere S, Chigbu B, Okoro O, Kamanu CI, Aluka C, Onwere A. Knowledge of genital herpes infection among antenatal clinic attendees in South-Eastern Nigeria. East Afr Med J. 2009; 86(7): 344-7.

16. Nath A K, Thappa DM. Newer trends in the management of genital herpes. Indian J Dermatol Venereol Leprol. 2009; 75(6): 566-74.

17. Mertz GJ. Asymptomatic Shedding of Herpes Simplex Virus 1 and 2: Implications for Prevention of Transmission. J Infect Dis [Internet] 2008 Oct [citado 10 octubre 2010]; 198(15): 1098-1100. Disponible en: http://www.journals.uchicago.edu/doi/pdf/10.1086/591914

18. Warren T, Ebel B. Counseling the Patient who has Genital Herpes or Genital Human Papillomavirus Infection. Infect Dis Clin North Am [Internet] 2005 Dic [citado 20 noviembre 2010]; 19(2): 459-476. Disponible en: http://www. sciencedirect.com

19. Dibble SL, Swanson JM. Gender differences of the predictors of depression in young adults with genital herpes. Public Health Nurs. 2000; 17(3): 187-194.

20. Bruce MK, McLaughlin J. The development of the scale to assess knowledge and attitude about genital herpes. The journal of sex research. 1986; 22(1): 73-84.

21. Guillemin F, Bombardier C, Beaton D. Cross cultural adaptation of health related quality of life measures: Literature review and proposed guidelines. J Clio epidemiol [Internet] 1993 Feb [citado 2 febrero 2008]; 46(12). Disponible en: http://www.jstor.org/stable/4034559

22. Moriyama IM. Indicators of social change. Problems in the measurements of health status. New York: Russel Sage Foundation; 1968. p. 593.

23. Squire VE. Cuba consejería. Multimedia sobre consejería [CD-ROOM]. Primera edición. Ciudad Habana: CNP ITS-VIHSIDA; 2007.

24. Gilbert L, Wyand F. Genital Herpes Education and Counselling: Testing a Onepage 'FAQ' Intervention. Herpes. 2009; 15(3): 51-5.

25. Abreu LM, Peña TR, Fiol JJ, Gómez AE, Daniel AA, Cárdenas LZ, et al. Infecciones de transmisión sexual, pautas para su tratamiento. Ciudad Habana: MINSAP PNUD; 2004.

26. Sánchez RE. La infección por herpes simple genital en el municipio Centro Habana. 2003-2007 [Tesis maestría]. Cuba: Centro Habana, Hospital General Docente Calixto García Iñíguez; 2008.

27. García SN. Llaman a usar nuevos métodos contra infecciones sexuales. Juventud Rebelde. 13 May 2010; Cuba: 5.

28. ITS/VIH/SIDA [sede web]. Centro de 
Información Científico- Centro Nacional de Prevención de ITS-VIH/SIDA- MINSAP: Lídice Mederos Villalón; 2009-2010 [Actualizado 20 Oct 2010]. Plan Estratégico Nacional ITS/VIH/SIDA 2007-2011 [89 páginas]. Disponible en: http://www. sld.cu/galerias/pdf

29. ITS/VIH/SIDA [sede web]. Centro de Información Científico- Centro Nacional de Prevención de ITS-VIH/SIDA - MINSAP: Lídice Mederos Villalón; 2009-2010 [Actualizado 27 Oct 2010] LíneAyuda [una pantalla]. Disponible en: http:// www.sld.cu/servicios/sida/
30. Rodríguez LR. Caracterización de conocimiento, actitud y uso del condón masculino en la población de riesgo de contraer VIH/SIDA. Cuba. 2005 [Tesis maestría]. Cuba: Escuela Nacional de Salud Pública; 2005.

31. González G, Hoyos M, Martínez L, González I, Lama A. Intervención educativa sobre ITS-VHI/SIDA en estudiantes de la enseñanza preuniversitaria. IPVC Carlos Marx. Años 2008-2009. Rev Med Electrón [Internet] 2010 Jul-Ago [citado 1 diciembre 2010]; 32(4): [6 p.] Disponible en: http://scielo.sld.cu 


\section{Anexo 1. Versión del instrumento para medir conocimiento sobre herpes simple adaptado} culturalmente al contexto cubano.

\section{Escala para el conocimiento de Herpes}

Código

Edad

Sexo

Estado Civil

Nivel de escolaridad

Para cada uno de los 51 planteamientos, responda si usted considera que el planteamiento es verdadero o falso, si usted no sabe si es verdadero o falso entonces responda que no sabe que la afirmación es correcta o falsa.

Use los siguientes códigos para las respuestas:

V VERDADERO.

F FALSO.

? NO CONOZCO SI ES CORRECTA O FALSA.

Cada planteamiento está numerado. Asegúrese que cada planteamiento enlace con su respuesta. Por favor, responda todos los planteamientos, no deje planteamientos sin responder. Le recuerdo que sus respuestas son estrictamente confidenciales.

\section{Planteamientos}

1. La duración y la severidad de las lesiones por Herpes Genital varían de persona a persona.

2. El Herpes Genital es causado por un virus.

3. El Herpes Genital se descubrió hace cinco años.

4. La menstruación (en el sexo femenino) o el coito pueden provocar la reaparición de los Herpes Genitales.

5. Toda persona que tiene un brote primario(por primera vez) tendrá una reaparición del mismo al año siguiente.

6. El Herpes Genital hace infértil (estéril) al hombre.

7. Entre las reapariciones el virus del Herpes permanece latente (inactivo) en las células nerviosas.

8. El Herpes tipo I no afecta los genitales.

9. Una persona que tenga reapariciones de herpes genital con frecuencia experimenta sensaciones prodrómicas (primeros síntomas).

10. Pueden pasar años entre la aparición de llagas por herpes.

11. Cualquier llaga en los genitales es un herpes.

12. Una vez que la llaga del herpes haya sanado, la persona no volverá a tener otra llaga a causa del herpes.

13. Las personas que tienen lesiones por herpes en la boca son inmunes a adquirir las lesiones en los genitales.

14. La lesión por Herpes Genital por lo general se presenta en forma de vesículas agrupadas.

15. Las sensaciones prodrómicas (primeros síntomas) de las apariciones de los herpes genitales incluyen cosquilleo y picazón en la zona donde éstos normalmente aparecen.

16. Si una persona tiene un contacto sexual con alguien que tiene Herpes indudablemente adquirirá la enfermedad también.

17. Se prevé que en el presente año varios cientos de personas adquieran herpes genital por sentarse en las tasas sanitarias.

\begin{tabular}{|c|c|}
\hline Verdadero(V) & Falso(F) \\
\hline $\mathrm{V}$ & $\mathrm{F}$ \\
\hline $\mathrm{V}$ & $\mathrm{F}$ \\
\hline $\mathrm{V}$ & $\mathrm{F}$ \\
\hline $\mathrm{V}$ & $\mathrm{F}$ \\
\hline $\mathrm{V}$ & $\mathrm{F}$ \\
\hline $\mathrm{V}$ & $\mathrm{F}$ \\
\hline $\mathrm{V}$ & $\mathrm{F}$ \\
\hline $\mathrm{V}$ & $\mathrm{F}$ \\
\hline $\mathrm{V}$ & $\mathrm{F}$ \\
\hline $\mathrm{V}$ & $\mathrm{F}$ \\
\hline $\mathrm{V}$ & $\mathrm{F}$ \\
\hline $\mathrm{V}$ & $\mathrm{F}$ \\
\hline $\mathrm{V}$ & $\mathrm{F}$ \\
\hline $\mathrm{V}$ & $\mathrm{F}$ \\
\hline $\mathrm{V}$ & $\mathrm{F}$ \\
\hline $\mathrm{V}$ & $\mathrm{F}$ \\
\hline $\mathrm{V}$ & $\mathrm{F}$ \\
\hline
\end{tabular}


18. La mujer que adquiera la infección por Herpes Genital quedará estéril o imposibilitada a tener hijos.

19. Las personas que usan lentes de contacto y tienen herpes labial deben evitar ponerse los lentes en la boca para evitar el contagio a los ojos.

20. A la persona que sufre de herpes genital se le orienta que debe mantener la zona de las llagas limpia y seca.

21. Los herpes genitales provocan la muerte

22. Existe cura para el Herpes Genital en estos momentos.

23. Por lo general, las reapariciones de herpes genitales no suelen ser menos frecuentes con el tiempo.

24. Una persona que sufre de herpes genital con frecuencia tiene más complicaciones psicológicas que físicas.

25. Cuando una persona tiene la lesión por Herpes Simple es recomendable no tener relaciones sexuales.

26. Las personas con Herpes Genital a veces pueden anticipar cuándo tendrán las reapariciones.

27. El Herpes Genital puede contagiarse aun si la lesión tiene costra.

28. El primer episodio de Herpes Genital es más severo que las reapariciones.

29. La persona que tiene Herpes Genital es inmune a los herpes orales (en la boca).

30. Entre las reapariciones el virus del Herpes Genital permanece inactivo cerca de la médula espinal.

31. Con frecuencia, el estrés puede desencadenar las reapariciones en los pacientes con Herpes Genital.

32. El Herpes es fatal para los recién nacidos si contraen la enfermedad.

33. Con frecuencia, los Herpes Genitales suelen ser detectados mediante la prueba citológica..

34. En una persona, después de haber estado expuesta al herpes genital, por lo general aparecen los primeros síntomas en un período de 2 a 20 días.

35. La ansiedad puede desencadenar las reapariciones en el Herpes Genital.

36. El uso del condón ofrece un 100\% de protección contra el Herpes Genital.

37. La infección por Herpes Genital generalmente conduce a la sífilis.

38. Una mujer que haya padecido de Herpes Genital debe hacerse cesárea si tiene un bebé.

39. La mayoría de las personas han estado expuestas al virus del Herpes Labial.

40. Una mujer con Herpes Genital puede dar a luz a su bebé si no tiene infección por Herpes Genital visible en el momento del parto.

41. Si ambos padres tienen Herpes Genital, sus hijos nacerán con Herpes también.

42. La mujer que tenga Herpes Genital nunca podrá tener hijos.

43. El Herpes Genital no es contagioso.

44. El Herpes Genital está asociado con el cáncer cérvico-uterino.

45. Los métodos anticonceptivos matan el virus del Herpes Genital.

46. En el primer episodio con Herpes Genital la persona puede sentirse como si tuviera catarro.

47. El Herpes Labial es contagioso.

48. La mejor forma de curar el Herpes Genital es manteniendo la lesión húmeda.

49. La persona con Herpes Genital no transmite el virus si está en la etapa de síntomas prodrómicos o síntomas de alerta.

50. El Herpes Genital no es común en las universidades.

\begin{tabular}{|c|c|}
\hline V & $\mathrm{F}$ \\
\hline V & $\mathrm{F}$ \\
\hline V & $\mathrm{F}$ \\
\hline V & $\mathrm{F}$ \\
\hline V & $\mathrm{F}$ \\
\hline V & $\mathrm{F}$ \\
\hline V & $\mathrm{F}$ \\
\hline V & $\mathrm{F}$ \\
\hline V & $\mathrm{F}$ \\
\hline V & $\mathrm{F}$ \\
\hline V & $\mathrm{F}$ \\
\hline V & $\mathrm{F}$ \\
\hline V & $\mathrm{F}$ \\
\hline V & $\mathrm{F}$ \\
\hline V & $\mathrm{F}$ \\
\hline V & $\mathrm{F}$ \\
\hline V & $\mathrm{F}$ \\
\hline V & $\mathrm{F}$ \\
\hline V & $\mathrm{F}$ \\
\hline V & $\mathrm{F}$ \\
\hline V & $\mathrm{F}$ \\
\hline V & $\mathrm{F}$ \\
\hline V & $\mathrm{F}$ \\
\hline V & $\mathrm{F}$ \\
\hline V & $\mathrm{F}$ \\
\hline V & $\mathrm{F}$ \\
\hline V & $\mathrm{F}$ \\
\hline V & $\mathrm{F}$ \\
\hline V & $\mathrm{F}$ \\
\hline V & $\mathrm{F}$ \\
\hline V & $\mathrm{F}$ \\
\hline V & $\mathrm{F}$ \\
\hline V & $\mathrm{F}$ \\
\hline V & $\mathrm{F}$ \\
\hline
\end{tabular}
les. 